\title{
Enhancing utilization of the findings from the youth reproductive health project in Senegal
}

Nafissatou J. Diop

Population Council

Anta Fall Diagne

Population Council

Follow this and additional works at: https://knowledgecommons.popcouncil.org/departments_sbsr-rh

Part of the Health Services Research Commons, International Public Health Commons, Public Health Education and Promotion Commons, and the Social and Behavioral Sciences Commons How does access to this work benefit you? Let us know!

\section{Recommended Citation}

Diop, Nafissatou J. and Anta Fall Diagne. 2008. "Enhancing utilization of the findings from the youth reproductive health project in Senegal," FRONTIERS Final Report. Washington, DC: Population Council. 


\title{
Enhancing Utilization of the Findings from the Youth Reproductive Health Project in Senegal
}

\author{
Nafissatou J. Diop, Ph.D \\ Dr Anta Fall Diagne
}

March 2008

This study was made possible by the generous support of the American people through the United States Agency for International Development (USAID) under the terms of Cooperative Agreement No. HRN-A-00-98-00012-0. The contents are the responsibility of the FRONTIERS Program and do not necessarily reflect the views of USAID or the United States Government 


\section{SUMMARY}

In 1999, FRONTIERS began a three-year collaboration with the World Health Organization (WHO), the Senegalese ministries of Education (MOE), of Youth (MOY), and Family and Social Affairs (MOFSA), the Center for Research and Training (CEFOREP) and the Population Training Group (GEEP) to test the feasibility, effectiveness and cost of school-, clinic-, and community-based interventions to improve the reproductive health of youth aged 10-19 years. The Operations Research (OR) study focused on providing information appropriate to the cultural context of Senegalese adolescents on a variety of reproductive health issues including: knowledge of the human body; puberty; sexuality; gender roles; different cultural, familial, and peer values regarding sexual and reproductive behavior; contraception; responsible parenthood; STIs and AIDS; and sources of help and services.

The study tested different ways of providing information. At the community through outreach, an 'edutainment' format was adopted; this comprised interactive participation of beneficiaries involving both education and entertainment. In schools, extra curricular activities and events such as music festivals were used as the forum. In clinics, a combination of edutainment and standard approaches of providing information were used.

The study was undertaken in three urban communities in northern Senegal. It had an overall positive result on young people's reproductive health knowledge, enhanced community understanding of youth reproductive health needs, and showed that a multisectoral approach is vital to supporting young people's healthy growth (See FRONTIERS OR Summary 35). This success prompted a follow-on effort, beginning in 2004, to institutionalize youth reproductive health in the study districts, create a favorable policy and funding environment for adolescent reproductive health at the national level, and scale up the intervention in Senegal and to other Francophone African countries (Mauritania, Guinea, Burkina Faso, Mali). This summary describes the essential process elements of this institutionalization effort.

FRONTIERS provided technical assistance to the MOE, MOY, MOFSA, youth-serving organizations and development partners to reinforce their capacity to develop and implement measures to improve adolescent reproductive health. Through the MOH, FRONTIERS supported training for service delivery organizations to increase their ability to develop and implement multisectoral interventions. Technical assistance (TA) was provided to the government ministries develop action plans which were submitted to development partners for funding.

\section{Results}

In 2004, the MOH established a multisectoral steering committee to lead development of an inter-ministerial coordination body to guide reproductive health activities. This committee was created to follow-up recommendations from the dissemination of the OR study results. It is composed of thirteen member organizations representing the government, NGOs and development partners (Ministry of Health's RH Division, WHO, Population Council, UNFPA, MOY, Projet Promotion des Jeunes, Center for Training and Research in Reproductive Health (CEFOREP), Groupe d'Etude et d'Enseignement pour la Population (GEEP), Association Senegalaise pour le Bien Etre Familial (ASBEF), UNICEF and GTZ). The MOH Reproductive Health division led the process of institutionalization and scaling up. 


\section{Policy Impact}

\section{Development of a policy document on Strategic Orientation for Adolescent Health}

FRONTIERS supported the MOH Reproductive Health Division (DSR in French) to produce an innovative paper on Strategic Orientations on adolescent health. This followed up on a number of recommendations: to integrate the activities demonstrated in the OR study into on-going activities of the ministries and NGOs; to extend the lessons learned throughout the regions; to increase financial support by locally elected committees to maintain adolescent reproductive health (ARH) programs; and to reinforce capacity of and assist ministries of Health and Youth in developing and putting into effect measures to improve ARH. This paper, which served as a national guide for program leaders both at the national and the decentralised local levels, defined both general and specific objectives, guiding principles, main strategies, priority activities, plans of action, an institutional framework and monitoring and evaluation systems for adolescent health. This paper was based on the findings from the OR study and was the result of a long process, in which the partners on the steering committee, which was expanded to include other partners such as WHO, CEDPA and other ministerial sectors, development partners and NGOs, all took part. This document was approved in June 2005 by a group of experts in reproductive health and youth issues. The approval process included participation by a representative of the Ministry of Health from each of the 11 Regions in Senegal, several departments of the Ministry of Education, several departments from the Ministry of Youth, the STI/AIDS Division, the Ministry of Planning and Sustainable Development, the Ministry for Social Development, GEEP, CEFOREP, ASBEF, UNFPA, UNICEF and MSH.

\section{Inclusion of Adolescent Reproductive Health in RH Norms and Standards}

The five-yearly revision of the Norms and Standards for Reproductive Health, conducted by the Ministry of Health in December 2004, provided an opportunity to include the issue of ARH in these documents for the first time. The lessons learned from the OR study greatly helped FRONTIERS, WHO and the DSR to propose a framework and procedures for ARH in Senegal, which included: a definition of adolescent health; aims and justification; main strategies; a set of services that adolescents should receive; expectations of the beneficiaries; norms in personnel, equipment and infrastructure; and the stages of consultation for service providers with adolescent clients.

\section{Establishment of Adolescent Health as a priority in the RH agenda}

The MOH was reorganized in 1999 when it prioritized adolescent reproductive health. As a result, improving the understanding of adolescent reproductive health issues and challenges has become a priority for the DSR. With FRONTIERS support during the February 2005 USAIDsupported Conference on Repositioning Family Planning, the Ministry of Health and its partners insisted that improving adolescent reproductive health would have the greatest impact on overarching goals to increase contraceptive prevalence and reduce maternal mortality in Senegal. The Ministry stressed that adolescent behavior is more susceptible to change than that of adults and that youth who practice family planning are more likely to continue use into their adult lives.

\section{Development of tools to support policy}

The Ministry of Health, with the support of FRONTIERS, began the extension of its activities into the other Regions in Senegal by creating standardized technical forms on adolescent health 
to guide activities at the District level. Thirteen technical forms were developed, one on each of the following:

1. Identification of service delivery points for providing youth friendly services

2. Identification of staff in need of training;

3. Training of health providers;

4. Facility self-evaluation:

5. Facility reorganization to address adolescent needs;

6. Identification of youth organizations;

7. Selection of peer educators;

8. Training of peer educators;

9. Equipment provision;

10. Peer educator supervision systems;

11. Organization of radio programs;

12. Training members of the technical committee;

13. Implementation of $\mathrm{BCC}$ and promotional activities.

A technical form is a one-page document, which contains the objective of the activity, the target to be achieved, who will carry out the activity, the cost and the length of the activity and how it is to be financed. These forms were distributed throughout Senegal, with the aim of encouraging Districts to include ARH activities into their operational plan for 2005. The operational plan is an annual exercise involving the District team, partners, NGOs and community representatives. After sending the forms, a follow up visit was usually made by the DSR and FRONTIERS to ensure that ARH activities have been properly included in the operational plans.

\section{Programmatic Impact}

\section{Adoption as official documents the 'Curriculum Grandir en Harmonie' and 'Orientation of} Health providers in Adolescent Health'. The curriculum developed by FRONTIERS during the OR study was very popular and has shown its effectiveness in improving adolescent health. Therefore the MOH RH division decided to use it for adolescent education nationwide. A revision of the tools by a group of multisectoral experts led to the official adoption of these documents as National training tools in June 2005.

Grandir en Harmonie is a life skills curriculum with a dynamic, interactive and participative approach for the training of peer educators with eight modules (Let's know each other, Values clarification, Knowledge of the body, Sexuality, Reproduction and pregnancy, Contraception, STI/HIV/AID, Responsible parenthood ). 
Capacity Building of $\mathrm{MOH}$ and partners trainers. A national pool of trainers and regional pools of trainers were trained by FRONTIERS with the two curricula Grandir en Harmonie and Orientation of Health providers in Adolescent Health. Three training of trainers (TOT) sessions were organized by the Adolescent Office of the $\mathrm{MOH}$, with technical assistance from FRONTIERS. The two national training curricula for health providers and for peer educators were used for the TOT. About 100 master trainers are now available at both central and regional levels, including youth representatives, $\mathrm{MOH}$ regional and district management teams, agents from ministries of Youth, Education, Social Affairs, and development partners. Following these TOTs, a cascade of training for health providers took place in eight regions, training 120 health providers (15 per region) in the Orientation Program for Health Providers on Adolescent Health.

Consolidation and sustainability in the two health Districts of Saint-Louis and Louga. A situation analysis undertaken recently showed that the ARH activities are still sustained in the original two districts since the end of the OR study in 2003. The district health teams remain a driving force. Re-training, supervision visits and planning activities were done by FRONTIERS and the Reproductive Health Division in order to consolidate and sustain the ARH activities.

Dynamic role of peer educators. FRONTIERS built the capacity of youth organizations following the model proven successful during the OR study for strengthening peer educators. The peer educators are today major actors in the two pilot districts. Out of 70 peer educators initially recruited during the OR study in both sites, 57 were still present within their youth association; the 13 peer educators that had left, most of them girls, did so because of marriage or job opportunities. The youth associations took responsibility for replacement of the departed peer educators, since the end of the OR study, about 20 new peer educators have been recruited to replace those who have left. Not only did they remain keen and active, but following a suggestion from FRONTIERS staff, they were inscribed as members of an official organization that is registered with the police structure of the Department and with a document endorsed by the Governor. The senior staff of the Health District and the coordinator of the youth center at Louga supervise their activities.

These associations gained recognition from other national organizations and development partners present in the region and were well accepted by the adolescent community itself, through helping to refer people to medical or psychosocial resources. With the name "Association des Aides Ados et Pairs Educateurs en Santé de la Reproduction" (Association of Adolescent Help and Peer Educators in Reproductive Health) in Saint-Louis, and "Association des Educateurs Communautaires de Santé" (Association of Community Educators) in Louga, both associations had offices, one at the Health Center in Saint-Louis and another in the Departmental Center for Popular and Sport Education (CDEPS) of the Ministry of Youth in Louga. The members of the associations kept up their links with their own community-based organizations. With guidance from FRONTIERS, the associations also maintained close links with the Health District, which involved them systematically in the activities of other campaigns, such as those aimed at preventing malaria, tuberculosis and HIV/AIDS, and promoting immunization. This enabled the District to maintain contact as well as giving them some economic incentive and training. Their abilities and knowledge were often sought after by other development partners, such as Plan International, FHI and MSH. The two associations received financial support in 2005 from the National AIDS Control Program (CNLS) and the Global Fund through an NGO, the Agence pour la Promotion des Activités de Population (APAPS). In addition, each member of the association contributed FCFA 1,000 every month to a common 
fund. Based on the needs expressed during the situation analysis, refresher training, discussions and planning were carried out by FRONTIERS and the RH division.

Effective strengthening of partnerships. During the OR study, FRONTIERS established a partnership between the Health District, the ministries of Youth and of Social Development, the NGO Action Educative en Milieu Ouvert (AEMO) and youth associations. In Louga, a strong partnership also existed between these organizations and the Youth Center. An example of this synergy in action was the establishment of a system for medical management of sexual assault (District), from a psychosocial point of view (Youth Center) and from a legal perspective (AEMO). This partnership is still ongoing.

\section{Utilization by the Ministry of Youth}

The Youth Promotion Project (PPJ) contacted FRONTIERS to request assistance for a meeting with local elected leaders to seek financing for its activities. FRONTIERS used the results of the research to prepare an advocacy presentation that has been used successfully by the PPJ. The PPJ, together with the Youth Directorate, is also using the curriculum "Grandir en Harmonie" as a basic tool for the training of Peer Educators for its 13 Youth Centers throughout Senegal. In addition, a presidential initiative is establishing "Youth spaces" at regional, departmental and rural level with the mandate to provide leisure, entertainment, communication and information technologies and RH classes. To date, eight youth spaces have been built.

\section{Utilization by the Ministry of Justice}

The Grandir en Harmonie curriculum is also being used by CLAIRE ENFANCE, a faith-based organization in Saint Louis that requested technical assistance from FRONTIERS for using the curriculum in their sensitization activities with vulnerable adolescents (e.g. house staff, shoeshine boys, car washers, teenage mothers, youth in prison). These groups of vulnerable youth are different than those generally reached by peer educators, and so CLAIRE ENFANCE and AEMO have adopted the teaching approach to be more appropriate for this audience; Following FRONTIERS training of peer educators, more than 400 adolescent boys and girls have already been reached through education sessions. A partnership was also created between AEMO and an American NGO called "Youth, Education and Sport" (YES), in which the curriculum is used during its holiday camps in Senegal.

\section{Full acceptance by the Ministry of Education of RH within schools}

The Ministry of Education and its NGO partner, GEEP, have fully integrated reproductive health as a key component to be included in school curricula. This is being gradually expanded and is anticipated to be sustainable based on FRONTIERS' efforts to disseminate the lessons learned, and to make sure that the Ministry of Education and GEEP participated in all the utilization activities at the central and local levels. The participation of the MOE and GEEP in strategic orientations and in curriculum revision enabled the momentum and the will to be maintained for this multisectorial and multidisciplinary approach.

GEEP, which was responsible for the school intervention during the OR study, continues to introduce and teach the curriculum for adolescent health. This curriculum, "le Devenir Accompagné" (A Guide for the Future), was introduced into two high schools in the Region of Thiès (at Thiès and at Mbour) and one high school in the Region of Louga (Dahra) during 2004/2005. A second phase of scale-up was then implemented at the high school des Parcelles Assainies in Dakar and at Mboro during 2005/2006. The curriculum "Grandir en Harmonie" is 
used for educational activities outside schools, such as vacation camps, with the help of other teaching aids, such as the manual "Brisons le Silence" (Let's Break the Silence).

Another GEEP project is the inclusion of reproductive health services in schools, with the development of a "school health space." Following experience with the OR study, GEEP recognized the importance of building bridges with health services and so developed a model that offers a package of services by a multi-disciplinary team (including a health worker) and education sessions. A computer room and a reading room will be included in this school health space. The pilot phase for this project has started recently in 12 high schools.

FRONTIERS has assisted the Division of Medical Schools to include reproductive health services in school health facilities by training school nurses to use the curriculum and by increasing awareness among parents and students. They have used the facility-based model developed during the OR study for reorganizing services to make the facility more attractive for adolescents.

\section{Integration of ARH by the Ministry of Sport}

FRONTIERS supported the Ministry of Sport to incorporate ARH into its training of sports instructors by training 25 sports teachers. The Ministry of Sport plans to strengthen the capacity of its staff to use the 'Grandir en Harmonie' curriculum for educating youth participating in their soccer camps.

\section{Scaling up in nine Regions of Senegal}

Leveraging support. With technical assistance from FRONTIERS, the MOH RH Division developed operational plans for ARH activities, including advocacy and capacity building, in the remaining nine regions of the country where the OR study had not been implemented. Funding from the World Bank IDA was leveraged to support these activities.

Integration of three ARH indicators in the Management Information System. During the process of harmonization of RH indicators, FRONTIERS assisted the DSR in the integration of three indicators of ARH in the MIS:

- Number of youth friendly health facilities;

- Number of adolescents attending health facilities;

- Number of adolescents going through life skills capacity-building.

Development of BCC materials. In scaling up ARH activities in Senegal, promotional tools were developed to help implementation of BCC activities. FRONTIERS assisted in the development of five posters, of which 5000 copies were produced, with funding from UNFPA, and widely disseminated among youth CBOs, health facilities and schools.

\section{Scaling up outside Senegal}

A national strategic plan for adolescent reproductive health was developed in Guinea with technical assistance from FRONTIERS and the Senegal MOH. The MOH authorities at the highest level in Guinea took a strong leadership role in the development of this policy document.

Translation of the curriculum 'Grandir en Harmonie' into Arabic. The Ministry of Education in Mauritania requested FRONTIERS assistance to adapt and translate into Arabic the 'Grandir en Harmonie' curriculum, with financial support from UNICEF and UNFPA. 


\section{Influencing other countries through a regional strategic orientation guide}

FRONTIERS and WHO jointly organized a regional consultation on community based interventions to improve the use of health services by adolescents on February 27-29, 2008 in Accra Ghana. The objective was to build consensus on strategic approaches to improve the participation of communities in the development and implementation of youth friendly health services. The consultation was an opportunity for FRONTIERS to: 1) share different lessons learned from implementation of the OR studies and their scaling up in Kenya and Senegal; and 2) incorporate FRONTIERS experiences in a document of guidelines on community based interventions to support youth friendly health services in Africa, which is currently under development. A total of 35 participants participated from ministries and NGOs in Senegal, Mauritania, Burkina Faso, Kenya, Tanzania, Mozambique, Namibia, Zimbabwe, and Ghana as well as staff from UNICEF, WHO, and IPPF.

\section{Utilization of OR Results by Development Partners and Technical Assistance Organizations}

Management Sciences for Health (MSH): trained school inspectors in 2003 with the curriculum developed during the OR study, "Le Devenir Accompagné." A total of 244 inspectors were trained in reproductive health with this curriculum. In addition MSH used the peer educators association for educational activities on maternal health and FP.

UNICEF Regional Office: the office recommends and makes use of the Grandir en Harmonie curriculum in other countries of the sub-Region, especially Burkina Faso and Benin. This office organized a regional workshop on "Young People and HIV/AIDS" in Dakar in 2005, bringing together the 15 countries covered by the office. FRONTIERS staff introduced the model of youth-friendly services.

Center for Development and Population Activities (CEDPA): in collaboration with FRONTIERS, and drawing from the results of the OR study, CEDPA has pilot-tested a model to strengthen parent-child communication in Senegal.

UNICEF and UNFPA: The questionnaire developed by FRONTIERS during the OR study served as the model for evaluating a joint UNICEF-UNFPA project for reinforcing the capacity of poor adolescents.

Save The Children Sweden uses the Grandir en Harmonie curriculum and the youth-friendly services model in its regional program in 18 countries.

Youth-serving organizations. FRONTIERS provided technical assistance to train trainers for the Christian faith-based organization, the YMCA, which has national coverage in Senegal. With funding from the World Bank the YMCA is implementing ARH activities through 200 youth networks in all 11 regions of Senegal. Another youth-serving organization, Synergie Banlieue, received technical assistance from FRONTIERS to implement their education activities within the region of Dakar. 


\section{Enabling Factors}

Several conditions enhanced utilization of the OR study results by the public sector and NGOs and mobilized public support for the ARH activities in Senegal.

- Multisectoral links. The involvement of multiple government agencies and community sectors established the importance of adolescent reproductive health as a broad social and policy concern. Their strong endorsement from the beginning gave further impulse to the multisectoral approach. Regular meetings organized by the Division of Reproductive Health brought together other sectors and development partners to plan and suggest activities. This was facilitated by the creation of a committee to follow up on the recommendations of the OR study.

- Evidence based results. Findings from the pilot operations research study were key for the institutionalization activities that followed because they had a scientifically proven basis. They also addressed a publicly recognized problem of adolescent reproductive health in Senegal. In addition, the ministries themselves were involved since the beginning.

- Use of existing institutions. One of the guiding principles of the OR study was to build sustainability through existing institutions, rather than establishing new structures. A key example of this was the partnership between youth associations and the public-sector health facilities, which resulted in unusually high commitment and retention of peer educators, as the health district engaged them in its maternal and child health activities. Today, the majority of the peer educators trained during the pilot project are still serving as trainers for other peer educators.

- Long-term investment. Addressing ARH required significant shifts in policy and social norms, which occurred over a period of several years. A major contributing factor in the project's sustainability and impact was the continuous support from USAID, which permitted follow up of the pilot sites, re-training of agents, development of plans and promotional tools to guide health districts and, most importantly, the availability of sustained and high quality technical assistance.

- Commitment of communities. The public recognized of adolescent reproductive health as a problem led parents to request more information on $\mathrm{RH}$ so that they could play a bigger role in their children's lives and establish stronger communications with them. Involvement and commitment of Youth-serving and community organizations facilitated these results.

- Involvement of government structures since the beginning. Even though the process was necessarily slow, the involvement of key government ministries and development partners has been key.

- Leadership by the MOH. Providing assistance to build the capacity of the MOH RH Department has proved extremely important to enable it to provide overall leadership of this process. 


\section{Hindering Factors}

Mobility of personnel. The mobility of personnel in government ministries, marked by their rapid and frequent turnover, slowed down the process. To institutionalize ARH in ministries, schools and health facilities is more effective if the staff can remain constant for sufficient time for the new activities to be introduced and become routine.

Lack of funding during the first two years to support and scale up the technical assistance supported by USAID through FRONTIERS initially slowed down the process of scaling up. Even today, resources are still scarce for adolescent reproductive health in Senegal, but the changes made in policy and programming achieved mean that it should be easier to ensure greater resources are allocated in the future, at national and regional level.

\section{Conclusion}

Utilization of the results from the OR study has led to an increase in the interest in and dynamism of key stakeholders for adolescent health in Senegal. All the policy documents and tools developed and the participatory process of implementation contributed to ownership by the various government sectors and by youth organizations, thereby creating a solid basis for sustainability. The most important recommendations for others seeking to replicate this successful approach to maximizing utilization and scale up of findings from research are the continued involvement of key institutions right from the outset, use of a participatory process to ensure ownership and build capacity, and widespread and repeated dissemination of the results expressed as programmatic recommendations. 


\section{REMERCIEMENTS}

Tous nos remerciements vont à l'encontre de l'USAID qui a bien voulu accepter de soutenir le processus d'utilisation des résultats de la recherche opérationnelle durant 3 années.

L'équipe adresse aussi ses vifs remerciements à toutes les personnes qui se sont investies dans la réalisation de ce travail. Leur engagement a contribué à son succès. Il s'agit d'abord du Ministère de la Santé et de la Prévention Médicale à travers sa Division de la Santé de la Reproduction, ses équipes cadres de Région et de Districts et aussi le Ministère de la Jeunesse, de la Femme, de la Famille et du Développement Social, et de l'Education.

L'exécution du projet a été facilitée par l'appui et la disponibilité d'institutions et de personnes qui ont composé le Comité restreint chargé du suivi des recommandations des activités de santé des adolescents.

Ce projet a aussi bénéficié de l'appui d'experts dans le domaine de la santé et du développement des adolescent(e)s/Jeunes.

Cette liste n'est certainement pas exhaustive, plusieurs autres personnes ayant apporté une précieuse contribution pour la réalisation de ce travail. Qu'elles trouvent ici l'expression de notre sincère reconnaissance. 


\section{TABLE DES MATIERES}

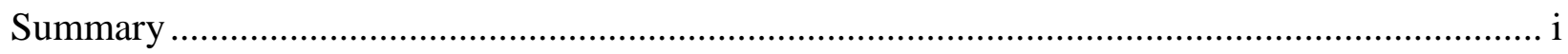

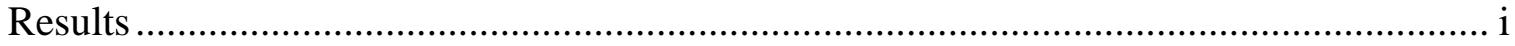

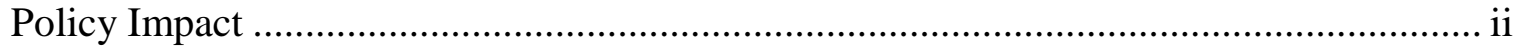

Programmatic Impact ............................................................................................... ii

Utilization of OR Results by Development Partners and Technical Assistance

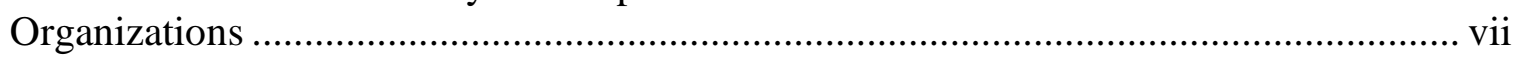

Enabling Factors..................................................................................................... vii

Hindering Factors .............................................................................................. ix

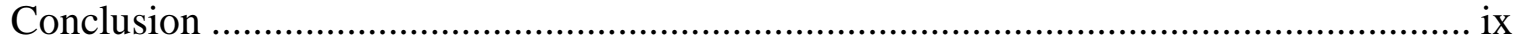

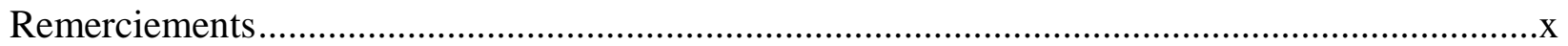

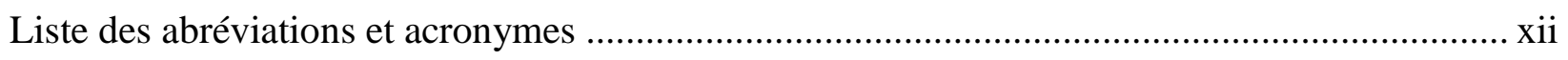

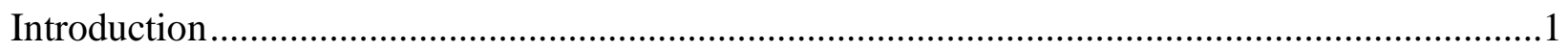

Contexte de la Santé des Adolescents ...............................................................................1

Les interventions mises en œuvre ............................................................................

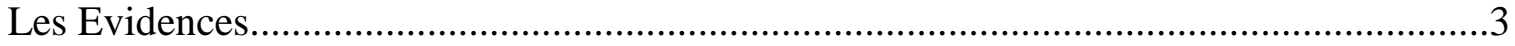

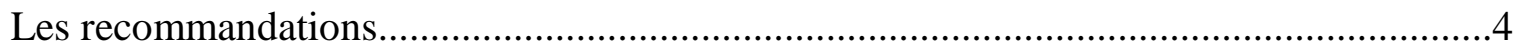

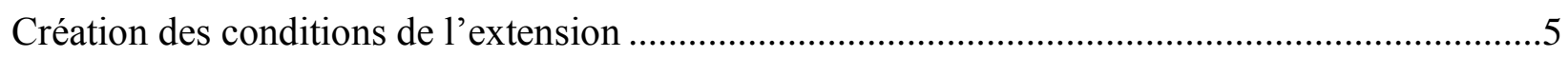

Processus mis en place pour une meilleure utilisation des résultats ....................................6

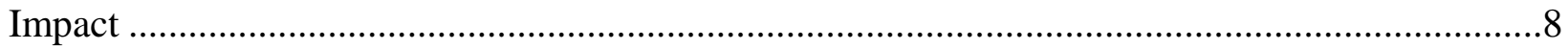

Institutionnalisation de la Santé des Adolescents au niveau du Ministère de la Santé et de la Prévention Médicale ...............................................................................

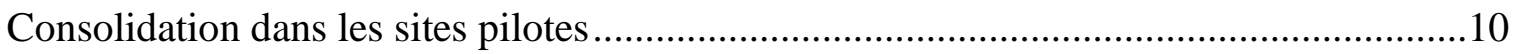

Intégration de la Santé des Adolescent(e)s dans les autres secteurs ................................13

Extension dans les autres régions du Sénégal .................................................................14

Utilisation d'outils élaborés ou des éléments du Modèle de la recherche opérationnelle par d'autres partenaires au développement ................................................16

Facteurs de réussite de l'utilisation des résultats de la recherche opérationnelle ..............19

Facteurs contextuels ayant limité l'utilisation des résultats de la recherche......................20

Conclusion 


\section{LISTE DES ABREVIATIONS ET ACRONYMES}

$\begin{array}{ll}\text { DSR } & \text { Division de la Santé de la Reproduction } \\ \text { CEFOREP } & \text { Centre de Formation et de Recherche en Santé de la Reproduction } \\ \text { COPE } & \text { Client-Orienté/Prestataire-Efficace } \\ \text { OMS } & \text { Organisation Mondiale de la Santé } \\ \text { CCA } & \text { Centre Conseil pour Adolescent(e)s } \\ \text { CCC } & \text { Communication pour un Changement de comportement } \\ \text { PPS } & \text { Points de Prestation de Services } \\ \text { IST } & \text { Infection Sexuellement Transmise } \\ \text { CDEPS } & \text { Centre Départemental d'Education Populaire et Sportive } \\ \text { AEMO } & \text { Action Educative en Milieu Ouvert } \\ \text { UNFPA } & \text { Fonds des nations Unis pour la Population } \\ \text { MSH } & \text { Management Sciences for Health } \\ \text { PPJ } & \text { Projet Promotion des Jeunes } \\ \text { ASBEF } & \text { Association Sénégalaise pour le Bien-être Familial } \\ \text { UNICEF } & \text { Fonds des Nations Unis pour l'Enfance } \\ \text { GTZ } & \text { Cooperation Technique Allemande } \\ \text { USAID } & \text { United States Agency for International Development } \\ \text { PO } & \text { Plan Opérationnel } \\ \text { CEDPA } & \text { Centre pour le Développement et les Activités de Population } \\ \text { FISA } & \text { Fondation International de Secours et d'Amitié } \\ \text { CRLS } & \text { Comités Régionaux de Lutte contre le SIDA }\end{array}$




\section{INTRODUCTION}

\section{Contexte de la Santé des Adolescents}

Dans le cadre de son programme global appelé FRONTIERS en Santé de la Reproduction, Population Council a lancé une série de recherches opérationnelles sur l'Amélioration de la Santé de la Reproduction des Adolescents dans 4 pays : le Bangladesh, le Kenya, le Mexico et le Sénégal. Au Sénégal, cette recherche a débuté en Octobre 1999 avec pour but de tester la faisabilité, le coût et l'impact d'un ensemble d'interventions dans la communauté, dans les services et dans les écoles.

La Recherche sur l'Amélioration de la Santé de la Reproduction des Adolescents avait pour objectifs spécifiques :

- D'établir une base empirique pour l'intervention et l'évaluation par un processus diagnostique afin de comprendre les questions de santé de la reproduction de la jeunesse dans chaque localité ciblée ;

- De déterminer la faisabilité, le coût et les effets d'une intervention au niveau de la communauté, visant des leaders d'opinion, des réseaux existants d'adultes et de jeunes, et conçue pour fournir des informations en matière de santé de la reproduction des adolescents ;

- De déterminer la faisabilité, le coût et l'effet de l'amélioration de la capacité des structures de santé de la reproduction existantes à recevoir les adolescents, de la volonté et de la capacité des prestataires de service à offrir des services de qualité aux adolescents ;

- De déterminer si une intervention au niveau de l'école apporte un effet supplémentaire sur l'amélioration de la connaissance, des attitudes et du comportement des adolescents en matière de Santé de la Reproduction.

\section{Les interventions mises en œuvre}

Plusieurs institutions ont travaillé en synergie afin d'exécuter les interventions à la fois au niveau national et local. L'étude a été menée au Sénégal en collaboration avec la Division de la Santé de la Reproduction (DSR) du Ministère de la Santé, le Centre de Formation et de Recherche en Santé de la Reproduction (CEFOREP), et le Groupe pour l'Etude et l'Enseignement de la Population (GEEP). Des partenariats ont été également créés avec le Ministère de l'Education, le Ministère de la Jeunesse et le Ministère de la Famille. La méthodologie de l'étude a utilisé un procédé quasi expérimental avec deux zones d'interventions (Saint-Louis, zone 1 et Louga, zone 2) et une zone de contrôle (Diourbel). Des enquêtes pré et post interventions ont été effectuées en Avril 2000 et Juillet 2002, ciblant essentiellement les adolescents garçons et filles de 10 à 19 ans, ainsi que leurs parents.

Rendre les services plus conviviaux. Le Ministère de la Santé a identifié neuf (09) Points de Prestation de Services (PPS) dans chaque district et, en effectuant un exercice de COPE, des actions ont été entreprises pour les rendre plus conviviaux pour les jeunes. Par exemple, une salle a été allouée soit à plein temps ou à temps partiel, pour les activités de Communication pour le Changement de Comportement (CCC). Les dispositifs pour recevoir des clients adolescents ont 
été réorganisés afin de leur permettre d'avoir un accès rapide et confidentiel aux prestataires. Trente deux (32) prestataires de santé ont été formés conformément au curriculum de l'Organisation Mondiale de la Santé (OMS) pour les prestataires de santé, pour une meilleure prise en charge des adolescents et des jeunes. Onze (11) jeunes employés «Aides Ado » ont été déployés dans les structures de santé pour accueillir, conseiller et orienter les adolescents vers les prestataires de santé. Des activités de CCC se sont tenues dans la structure de santé et dans le quartier.

Renforcer les compétences des jeunes dans la communauté. Les activités d'éducation des jeunes ont été menées par soixante (60) pairs éducateurs formés en technique de Communication pour le Changement de Comportement grâce à un curriculum d'éducation - divertissement, "Grandir en Harmonie". Les pairs éducateurs ont déroulé ce curriculum auprès de groupes d'adolescents des associations des quartiers. Ils ont par ailleurs mené des activités de masse et profité des événements pour sensibiliser les jeunes et leurs parents (festivals, semaines culturelles, thédébats). Par ailleurs, le personnel du district et les pairs éducateurs ont tenu des discussions de groupes et intervenu sur les ondes des radios locales en faisant passer des messages sur la Santé de la Reproduction deux à trois fois par semaine.

Rendre l'environnement plus favorable. Pour améliorer l'environnement, les adultes leaders religieux, administratifs et communautaires ont été davantage sensibilisés sur le programme au cours de séminaires, de réunions et de visites à domicile. Des discussions avec les parents se sont tenues dans le quartier pour accroître leurs connaissances et leur permettre de communiquer avec les jeunes. Des matériels de CCC et de promotion ont été développés et distribués pendant les interventions.

Education des adolescents à l'école. Le GEEP qui compte plus de dix ans d'expérience de travail dans les écoles, s'est servi d'une équipe multidisciplinaire pour développer un curriculum appelé le "Devenir accompagné". Etant donné la spécificité de l'environnement à l'école, ils ont pensé qu'un curriculum d'éducation - divertissement comme "Grandir en Harmonie," serait difficile à appliquer en salle de classe. La stratégie choisie par le GEEP a été de renforcer le rôle des enseignants en incorporant des thèmes de Santé de la Reproduction dans le curriculum régulier. Le curriculum a été élaboré pour les écoles primaires, secondaires et les lycées. Six (06) écoles ont été choisies pour le tester et treize (13) autres écoles ont exprimé leur intérêt à suivre l'expérience. Vingt six (26) enseignants affiliés au GEEP ont été formés et supervisés. Des activités hors de la salle de classe ont été menées avec les pairs éducateurs de l'école (camps de vacances...).

La popularité des pairs éducateurs communautaires dans le district de Saint-Louis a suscité des demandes de la part de certaines écoles de tenir des séances d'éducation en Santé de la Reproduction, avec l'accord de l'Inspection du Ministère de l'Education. Les pairs éducateurs communautaires ont tenu des séances dans dix huit (18) écoles avec le curriculum "Grandir en Harmonie," en utilisant l'heure de l'après-midi habituellement consacré aux "Sujets mal compris par les jeunes" dans les écoles primaires, et les classes d'économie familiale dans les écoles secondaires. 


\section{Les Evidences}

L'évaluation finale a montré que les interventions ont exposé les adolescents à des informations sur la Santé de la Reproduction dans les deux zones d'intervention 1 et 2. Les deux groupes d'âge ont été touchés à l'école. Les garçons ont reçu plus d'information que les filles. Hors de l'école, le groupe d'âge de 15 à 19 ans a été le plus largement atteint. Dans la zone 3, le site de contrôle, la proportion des adolescents ayant reçu des informations en Santé de la Reproduction indique que d'intenses activités à grande échelle ont été effectuées dans cette zone.

- Les parents étaient bien conscients des programmes de Santé de la Reproduction pour les adolescents après l'intervention, et la plupart du temps, leur approbation de ces programmes s'est accrue. L'approbation des femmes était plus élevée que celle des hommes ;

- Les membres de la communauté ont fortement cautionné l'amélioration de la Santé de la Reproduction des jeunes, mais ils ont exprimé des sentiments mitigés concernant la sexualité des adolescents. Les chefs religieux étaient d'avis que les parents devraient discuter ouvertement de questions de Santé de la Reproduction avec leurs enfants, mais les parents manquaient de connaissances pour le faire en toute confiance ;

- Les communications sur la Santé de la Reproduction, aussi bien de la part des parents que des adolescents, se sont fortement améliorées. Les adolescents ont cherché à obtenir plus d'informations des adultes et de sources qualifiées que de leurs amis. Cependant, une atmosphère familiale propice à la communication fait toujours défaut et en même temps, les attentes des adolescents ont considérablement augmenté ;

- Les connaissances des jeunes en matière de Santé de la Reproduction ont augmenté. L'analyse de ce qui est connu sur la puberté et les risques associés avec une sexualité précoce montre que les niveaux d'information sont généralement faibles. Le niveau de connaissance sur la puberté et les risques associés à la sexualité était plus élevé chez les adolescents exposés aux interventions, particulièrement ceux de 15 à 19 ans. Seule la connaissance du cycle menstruel n'a pas montré de différence dans les trois sites entre le début et la fin du programme ;

- La proportion des adolescents connaissant une ou plusieurs méthodes contraceptives a considérablement augmenté dans les zones 1 et 2 , mais non dans la zone 3 . Considérant la distribution selon l'âge et le sexe, on a noté dans la zone 1 une plus grande connaissance sur l'utilisation des méthodes, particulièrement le condom. Les attitudes vis-à-vis de l'utilisation de cette méthode se sont développées, indiquant une plus grande tolérance à l'endroit des adolescents célibataires ;

- Les connaissances sur le SIDA étaient assez répandues avant la mise en œuvre des interventions, mais celles-ci ont permis de les renforcer. Les autres Infections Sexuellement Transmissibles (IST) étaient moins bien connues. Avant l'intervention, on estimait qu'un adolescent sur 10 dans les trois zones de l'étude connaissait l'existence des autres IST. Après l'intervention, les connaissances ont considérablement augmenté dans les zones 1 et 2. Dans la zone 3, la situation est restée presque la même ;

- L'activité sexuelle des adolescents est plus une affaire de garçons que de filles. Elle survient le plus souvent pendant la transition du groupe de 10 à 14 ans à celui de 15 à 19 ans. On a également noté que les adolescents éduqués avaient une plus grande tendance à 
avoir une activité sexuelle récente que ceux qui n'étaient pas éduqués. Par contre, les étudiants qui avaient des occupations scolaires et qui avaient été exposés à l'intervention étaient moins susceptibles d'avoir eu une activité sexuelle récente. Les facteurs liés aux croyances et pratiques religieuses et le fait de vivre avec les parents biologiques constituaient également des facteurs protecteurs. L'adoption de l'abstinence secondaire est plus élevée dans la zone 2 que dans la zone 3. Les adolescents de la zone 2 qui avaient déjà connu leur première expérience sexuelle avaient moins de chance de continuer que ceux de la zone 3 . Les adolescents de la zone 2 avaient de toute façon moins de relations sexuelles que ceux de la zone 3.

- Les adolescents sexuellement actifs ont eu une moindre utilisation des méthodes contraceptives à la fin du programme qu'au début. Cette baisse peut être expliquée par le fait que les adolescents avaient trouvé des partenaires sexuels plus réguliers, et il y avait par conséquent un risque moindre d'exposition aux IST/SIDA tandis qu'au commencement, il y avait davantage de partenaires occasionnels ;

- Après les interventions, la connaissance des structures sanitaires a augmenté dans les zones 1 et 3 mais non dans la zone 2, où le niveau de connaissance était déjà assez élevé. Les visites aux structures sanitaires étaient rares avant les interventions, et se situaient au même niveau dans les trois zones. A la fin des interventions, il y a eu une augmentation significative de ces visites, particulièrement dans les zones 1 et 3 .

Selon tous les indicateurs, les adolescents directement exposés aux interventions avaient de meilleurs comportements, connaissances et attitudes que les adolescents vivant dans les sites d'interventions sans avoir été exposés au programme pendant les 12 derniers mois de celui-ci.

Sauf pour la connaissance et l'utilisation de la structure sanitaire, les deux sites d'interventions, les zone 1 et 2, ont montré une plus grande amélioration que la zone 3, le site de contrôle.

\section{Les recommandations}

A la fin de l'étude, toutes les trois zones, Louga, Saint Louis et Diourbel, et également au niveau national, les Ministères de la Santé, de la Jeunesse, de l'Education et des Affaires Sociales ont recommandé l'extension des interventions. Par conséquent, le Ministère de la Santé a trouvé cette expérience très positive et a souhaité maintenir et étendre les modèles développés pendant l'étude de Recherche Opérationnelle. L'amélioration notée des connaissances, attitudes et comportements sexuels après seulement quinze (15) mois d'intervention est un bon indicateur qu'un réel impact pourrait être atteint avec des interventions à plus long terme avec les adolescents.

En outre, le personnel du district de Louga et Saint Louis et leurs partenaires (Centre Départemental d'Education Populaire et Sportive (CDEPS), Région Médicale, Action Educative en Milieu Ouvert (AEMO), Développement Communautaire), et les pairs éducateurs ont organisé un forum de deux jours pour discuter des activités générales et examiner les conditions nécessaires pour maintenir ce qui avait été accompli. Ils ont conclu que l'ensemble des activités ont contribué à:

- L'amélioration de l'éducation des adolescents en Santé de la Reproduction ;

- L'accroissement des prestations de services ; 
- Au Gain de popularité du curriculum "Grandir en Harmonie" dans la communauté et les écoles ;

- Un changement dans les attitudes des prestataires de santé. Ils démontraient à présent un plus grand intérêt dans l'encadrement et la Santé de la Reproduction des Adolescents. Ils ont même joué le rôle de médiateur avec les familles ;

- Une amélioration dans l'environnement des adultes parce que l'on a remarqué ni barrière, ni violente réaction hostile. Ceci a été accompli grâce au soutien accordé au travail des pairs éducateurs par les comités techniques locaux.

Les résultats de cette étude ont été largement disséminés au Sénégal, aussi bien au niveau central qu'au niveau local. En plus, des tables rondes de travail ont été organisées avec le Ministère de la Santé et le Ministère de la Jeunesse pour discuter plus profondément des stratégies, des leçons apprises et apporter des améliorations aux programmes d'intervention sur la Santé de la Reproduction des Adolescents.

Les disséminations ont été largement couvertes par les médias. Pendant trois (3) jours, un document de cinq (5) minutes a été diffusé à la Radio Télévision Sénégalaise (RTS). Les radios ont retransmis onze (11) émissions et les journaux ont publié environ une dizaine d'articles.

Pendant le séminaire de dissémination nationale, environ cent (100) participants provenant des ministères, ONG, bailleurs et associations de jeunes ont fait en autres les recommandations suivantes:

- L'intégration des activités de santé des adolescents dans les activités de routine et leur extension au niveau régional ;

- Le renforcement des connaissances des parents en matière de Santé de la Reproduction de sorte qu'ils puissent communiquer avec leurs enfants sur la Santé de la Reproduction et le $\mathrm{VIH} / \mathrm{SIDA}$.

\section{CREATION DES CONDITIONS DE L'EXTENSION}

Ces recommandations ont fait l'objet d'un projet pour faire le suivi des activités de santé des adolescents. Ce projet s'est effectué en deux temps. La première phase allant de Juin 2004 à Juin 2005 avait pour objectif de créer au Sénégal, les conditions permettant aux organisations travaillant dans les domaines de la jeunesse, de la santé et du développement de comprendre et d'utiliser les résultats de l'étude de Recherche Opérationnelle sur l'Amélioration de la Santé de la Reproduction des Adolescents. La deuxième phase du projet qui a duré de Juillet 2005 à Août 2007 a eu pour objectif de promouvoir l'intégration des leçons apprises et l'utilisation du modèle testé au Sénégal pour prendre en charge la santé de la reproduction des adolescent(e)s/Jeunes parmi la communauté des agences de collaboration, des bailleurs, des gouvernements et des Organisations Non Gouvernementales de l'Afrique de l'Ouest.

Ce projet a eu pour objectifs spécifiques de:

- Contribuer à la création d'un environnement favorable à l'extension des services en santé des Adolescents/Jeunes au Sénégal ; 
- Assister les Ministères de la Santé, de la Jeunesse et de 1'Education dans la planification et la mise en œuvre des programmes de Santé de la Reproduction des Adolescents ;

- Renforcer les capacités au sein des ministères de la santé, de la jeunesse et de l'Education en formation et mise en œuvre de programme de Santé de la Reproduction des Adolescents;

- Former un noyau de formateurs en santé des adolescents ;

- Renforcer les capacités des institutions d'offre de services pour l'extension d'interventions multisectorielles pour améliorer la santé des adolescent(e)s/Jeunes en Afrique de l'Ouest sur la base des leçons apprises de la recherche opérationnelle.

\section{Processus mis en place pour une meilleure utilisation des résultats}

Durant une année, des efforts ont été fournis afin de soutenir les activités de santé des adolescents dans les deux districts sanitaires de Saint-Louis et de Louga. Des activités ont été impulsées pour accompagner les différents partenaires dans le processus d'utilisation des résultats de la Recherche Opérationnelle sur l'amélioration de la Santé de la Reproduction des Adolescents au Sénégal.

\section{Concertation}

Des réunions avec la Division de la Santé de la Reproduction (DSR), bénéficiaire du programme ont permis de développer un plan d'action pour le suivi des recommandations.

\section{Création d'un Comité de Suivi}

Des rencontres avec le Directeur de la Santé et le chef de la DSR ont abouti à la création, par le Directeur de la Santé, d'un Comité de suivi des recommandations issues de la dissémination des résultats de la recherche en avril 2004. Ce comité est coordonné par le Ministère de la Santé. Il est composé de treize membres des structures gouvernementales, des ONGs et des partenaires au développement : la DSR, les autres départements du Ministère de la Santé, l'OMS, Population Council, UNFPA, MSH, PPJ, CEFOREP, ASBEF, UNICEF, GTZ. Ce comité a eu à orienter les actions, corriger les curricula de formation et autres outils de gestion et de supervision, amender la Stratégie Nationale en Santé des Adolescent(e)s/Jeunes au Sénégal et participer à la validation des documents.

\section{Implication des Partenaires au Développement}

Des réunions avec les partenaires au développement tels que USAID, OMS, UNFPA, GTZ ont permis de définir les stratégies et coordonner les efforts de mise en œuvre des activités de santé des adolescents. L'OMS a joué un rôle particulièrement important dans cette synergie. En effet, de par son rôle de conseiller du Ministère de la Santé, l'OMS a eu à conseiller les actions, organiser des réunions dans ses locaux, financer l'atelier de validation des outils et curricula, faire des visites des sites afin d'aider à la pérennisation des actions.

\section{Suivi des sites pilotes}

Des visites de terrain ont été effectuées dans les deux sites expérimentaux et des entretiens menés auprès des prestataires de santé, des pairs éducateurs, du personnel des Ministères de la Famille et du Développement Social, de la Jeunesse et les membres des équipes cadres des districts sanitaires avec des guides développés par quelques membres du comité de suivi. 


\section{Recyclage}

Des sessions de recyclages des comités techniques et des associations des pairs éducateurs ont été effectuées à Saint- Louis.

Des ateliers de dissémination de résultats ciblés ont été organisés auprès des Ministères de la Santé, de la Jeunesse (avec ses deux directions que sont la Direction de la Jeunesse et de la Vie Associative, DJVA et le Projet Promotion des Jeunes, PPJ).

\section{Révision et validation des outils}

Plusieurs ateliers de relecture et d'amendement des curricula de formation des prestataires de santé et Grandir en Harmonie pour l'éducation des adolescent(e)s/jeunes ont été organisés. Des nouveaux documents ont été élaborés afin de faciliter l'appropriation par les nouveaux acteurs. Il s'agit de la Stratégie Nationale en Santé des Adolescent(e)s/Jeunes au Sénégal et des documents annexes qui accompagnent le curriculum Grandir en Harmonie : le guide du facilitateur et les questions et réponses les plus fréquentes posées par les adolescents.

\section{Création d'outils de planification pour les districts sanitaires}

Afin de faciliter l'extension des interventions dans les autres régions et districts du Sénégal, le Ministère de la Santé avec l'appui de l'OMS et du programme FRONTIERS a créer des fiches techniques d'activités sur la santé des adolescents pour orienter les districts sur le processus de mise en œuvre d'un programme sur la Santé de la Reproduction des Adolescents. Ces fiches techniques, au nombre de quinze (15), portent sur : l'identification des Points de Prestation de Santé, l'identification de tous les personnels à former, la formation du personnel de santé, le COPE des structures de santé, la réorganisation des services, l'identification des associations de jeunes, la sélection des futurs pairs éducateurs issus des associations identifiées, la formation des pairs éducateurs sélectionnés, l'équipement des pairs éducateurs, leur supervision, l'animation d'émissions radio, le recyclage des membres du comité technique, le recyclage des pairs éducateurs et la dotation du comité technique en matériels CCC et promotionnels.

La fiche technique est un document d'une page contenant l'objectif de l'activité, la cible, les acteurs, le coût, la durée et la source de financement. Ces fiches sont envoyées dans toutes les régions du Sénégal dans le but d'encourager les districts à planifier des activités de Santé de la Reproduction des Adolescents dans les Plans d'Opérations (PO). Les plans d'opérations sont un exercice annuel de planification des activités de santé. Cependant un suivi doit être fait par la DSR pour s'assurer que des activités de Santé de la Reproduction des Adolescents ont effectivement été incluses dans le PO.

\section{Dissémination des résultats de la recherche}

Plusieurs ateliers de dissémination des résultats de la recherche opérationnelle sur l'amélioration de la santé de la reproduction des adolescents au Sénégal ont été organisés. 


\section{IMPACT}

L'impact s'est ressenti aussi bien au niveau des populations elle mêmes que sur la politique et les programmes de santé au Sénégal.

\section{Institutionnalisation de la Santé des Adolescents au niveau du Ministère de la Santé et de la Prévention Médicale \\ Intégration de la Santé de la Reproduction des Adolescents dans les Normes et Protocoles}

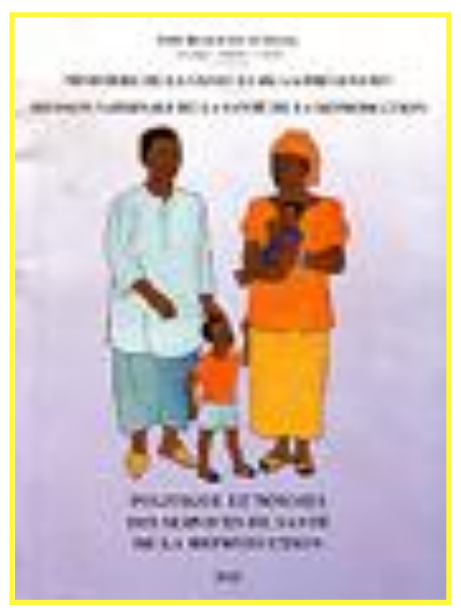

La révision quinquennale des Normes et Protocoles en Santé de la Reproduction, organisée par le Ministère de la Santé en décembre 2004 a offert une opportunité pour l'intégration de la Santé de la Reproduction des Adolescents dans ces documents pour la première fois. Les leçons apprises de la Recherche Opérationnelle, ont largement aidé FRONTIERS, l'OMS et la DSR à proposer un cadre et des procédures pour la Santé de la Reproduction des Adolescents au Sénégal incluant: les attentes des bénéficiaires, le but et le raisonnement, les principes directeurs, la définition des services de santé sexuelle et reproductive que les adolescents devraient recevoir, les points de prestation de services existants et les étapes dans une consultation que les prestataires de service devraient suivre pour l'adolescent.

\section{Priorisation de la Santé des Adolescents dans l'agenda de la Santé de la Reproduction}

Les leçons apprises de la Recherche Opérationnelle ont été d'une importance cruciale dans l'assistance apportée au Ministère de la Santé pour le développement d'un contenu sur la Santé de la Reproduction des Adolescents au Sénégal. L'appropriation de la Santé de la Reproduction des Adolescents par ce Ministère a permis de donner un contenu au mandat du bureau Santé des Adolescents créé en 1999 au sein de la Division de la Santé de la Reproduction.

La Santé de la Reproduction des Adolescents est devenue une priorité et durant la conférence de février 2005 sur le Repositionnement de la Planification Familiale, elle a été choisie par le Ministère de la Santé et ses partenaires comme un traceur pour l'amélioration de la Planification Familiale et la lutte contre la mortalité maternelle au Sénégal.

Depuis 2005, la Division de la Santé de la Reproduction intègre dans son Plan d'Opération (PO) des activités de santé des adolescents, telles que des ateliers de dissémination de l'expérience de Saint- Louis et Louga et de la Stratégie Nationale en Santé des Adolescent(e)s/Jeunes au Sénégal, des échanges d'expérience et l'appui aux régions et aux autres districts sanitaires. 


\section{Elaboration de la Politique Nationale de la Santé des Adolescent(e)s/Jeunes au Sénégal}

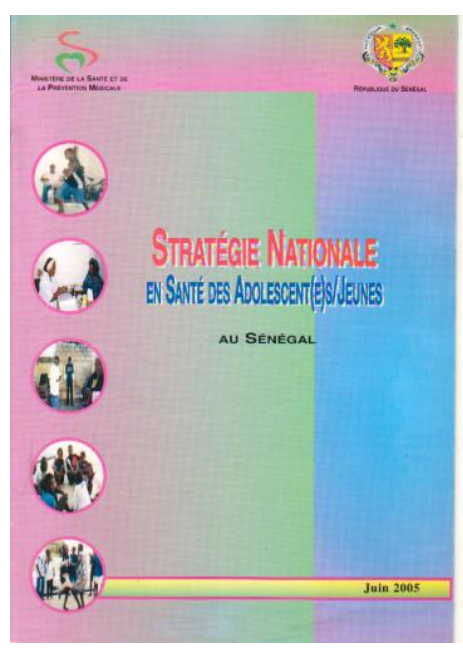

L'innovation apportée par le comité de suivi des recommandations aura été l'élaboration par la DSR, l'OMS et FRONTIERS, de la Stratégie Nationale en Santé des Adolescent(e)s/Jeunes au Sénégal qui constitue un outil national pour les responsables de programme au niveau central et décentralisé. Ce document définit les objectifs généraux et spécifiques, les principes directeurs, le cadre institutionnel, les activités prioritaires, les lignes d'actions et les mécanismes de suivi et d'évaluation des activités de santé des adolescents. L'élaboration de ce document s'est largement inspirée de la Recherche Opérationnelle et a été le résultat d'un long processus auquel ont pris part les partenaires du comité technique élargi à d'autres partenaires tels CEDPA des autres secteurs ministériels, des partenaires au développement et des ONGs. Ce document a été validé en Juin 2005 par un groupe d'experts en santé de la reproduction et en questions des jeunes. Etaient présents à l'atelier un représentant du Ministère de la Santé des 11 régions du Sénégal, la Division IST/SIDA, plusieurs départements des Ministères et de la Jeunesse, le Ministère du plan et du développement durable, le Ministère du Développement Social, le GEEP, le CEFOREP, l'ASBEF, l'UNFPA, l'UNICEF, MSH. Ce groupe, après examen de la Stratégie Nationale en Santé des Adolescent(e)s/Jeunes au Sénégal et des deux curricula de formation en santé des adolescents, a jugé que la qualité permettait d'en faire des documents nationaux de référence.

La Stratégie Nationale en Santé des Adolescent(e)s/Jeunes au Sénégal a été officiellement lancé par le Ministre de la Santé et de la Prévention Médicale en Septembre 2006 lors d'une cérémonie réunissant les autorités du Ministère de la santé et de la Prévention Médicale, des représentants d'autres secteurs ministériels (Jeunesse, Education Nationale, Femme, Famille et Développement Social, Collectivités Locales...), des partenaires au développement, des agences de coopération bilatérale et multilatérale, des réseaux et associations constitués, des organes de presse et des mouvements de jeunes.

\section{Renforcement de capacités des agents du Ministère de la Santé en Santé des Adolescents}

Une série de formation des formateurs en santé et développement des adolescent(e)s/jeunes a été entrepris au Sénégal par le Bureau des Adolescents du Ministère de la Santé et de la Prévention Médicale avec les deux curricula de formation destinés aux prestataires de santé et aux pairs éducateurs. Au total, 100 formateurs ont été formés y compris des prestataires de santé, des agents des Ministères de la Jeunesse, de l'Education, de la Femme, de la Famille et du Développement Social, des partenaires au Développement et des représentants d'OCBs de jeunes, notamment la section sénégalaise de l'ONG YMCA et Synergie Banlieue. 


\section{Consolidation dans les sites pilotes}

$\mathrm{Au}$ niveau local, l'analyse de la situation menée dans les deux sites expérimentaux de SaintLouis et de Louga a permis de mesurer l'impact programmatique de la recherche opérationnelle sur les activités de santé des adolescents.

\section{Consolidation et pérennisation des activités dans les deux districts sanitaires de Saint-Louis et Louga}

L'analyse de la situation a révélé que les activités de santé des adolescents sont toujours soutenues dans les points de prestations de services, dans la communauté et à l'école. Les structures de santé demeurent au centre du dispositif car elles intègrent effectivement les activités de santé des adolescents. L'observation des prestations de service dans les structures de santé de Louga et Saint- Louis a montré que les adolescents continuent à venir en consultation. Ils sont reçus, conseillés et, au besoin, orientés vers les prestataires de service. Ce constat est

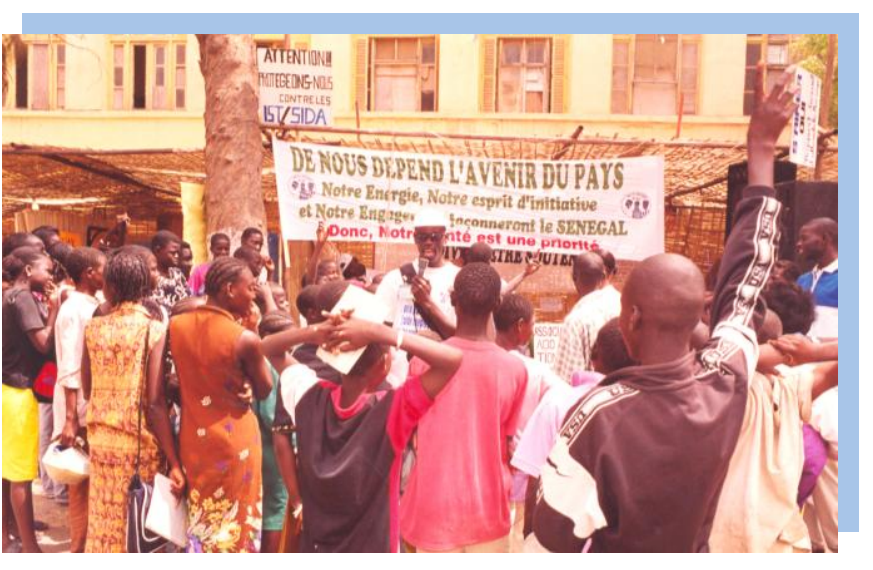
valable dans les deux districts sanitaires de Louga et Saint-Louis, d'autant plus que la plupart des salles ados qui avaient été aménagées au niveau des structures, fonctionnent encore. Les cinq salles ados de Saint-Louis ont conservé leur caractère convivial. Dans les centres de santé des deux villes de Louga et Saint-Louis et dans les autres postes de santé, les aides ados et les pairs éducateurs sont présents en grand nombre pour faciliter le circuit de l'adolescent. D'ailleurs le centre de santé de Saint-Louis sert de quartier général à l'association des pairs éducateurs. Dans les structures de santé, les images et les messages pouvant aider les adolescents à accepter la structure sont toujours disponibles sur les murs. De même, le circuit de référence de l'adolescent vers les prestataires de service continue d'être utilisé : les aides ados jouent un rôle déterminant dans ce processus en accompagnant les adolescents vers les prestataires de santé.

Il s'avère cependant difficile de donner des statistiques précises concernant le nombre d'adolescents fréquentant les structures de santé, car à la fin de l'étude, les outils de gestion permettant d'identifier la cible adolescente ont cessé d'être utilisés par les prestataires de santé. D'ou l'importance d'introduire des indicateurs sur la santé des adolescents dans le Système National d'Information (SNIS). Cependant, à Louga, 234 adolescent(e)s ont été reçus par les pairs éducateurs présents dans la structure et référés à l'équipe médico-sociale en place durant le deuxième trimestre 2005.

Le modèle de la recherche plaçant la structure de santé au cœur du dispositif semble mieux réussi à Saint-Louis qu'à Louga. L'engagement des prestataires de santé, de l'équipe cadre du district et des comités de santé à Saint-Louis est un élément fondamental à souligner. L'association des Pairs Educateurs communautaires bénéficie régulièrement du soutien financier du district sanitaire pour l'organisation des activités d'éducation envers les adolescents sur présentation d'un plan d'action. Comme exemple, dans l'année, ils mènent environ 4 grandes activités en tenant compte des évènements suivants : défilé de la fête de l'indépendance, festival de Jazz, Journée du 15 août, forum. Environ 2 millions de francs CFA leur sont attribués par le district chaque année et remis avant chaque activité. A Louga, les activités d'éducation sur la santé des 
adolescents sont plus soutenues par la municipalité que par le comité de santé du centre de santé. En effet, les autorités municipales de Louga se sont engagées dans les activités de SRA en construisant au centre de santé une salle ado. Par ailleurs, les pairs éducateurs travaillent en étroite collaboration avec le Centre Conseils Ado du Ministère de la Jeunesse.

\section{Dynamisme des pairs éducateurs}

Les pairs éducateurs constituent les piliers dans le cadre de l'utilisation des résultats de l'étude dans les deux districts. Sur 70 pairs éducateurs recrutés initialement dans les 2 sites, 57 sont encore présent dans les associations. Au total, 13 pairs éducateurs sont partis, la plupart des filles pour raison de mariage ou opportunité d'emploi. Le recrutement de nouveaux pairs éducateurs est fait par l'association des jeunes. Depuis la fin de l'étude 7 pairs éducateurs ont été recrutés pour remplacer les départs.

Non seulement ils demeurent toujours actifs et dynamiques, mais ils sont regroupés en une structure officielle avec un statut enregistré auprès de la préfecture de police du département, et un récépissé délivré par le gouverneur. Leurs activités d'animation dans les quartiers sont supervisées par les membres de l'équipe cadre du district sanitaire et le coordinateur du centre conseil à Louga. Cette association est reconnue par les autres organisations nationales et partenaires au développement de la région. Reconnus aussi par la communauté des adolescents, ils aident à référer vers les personnes ressources médicales ou psychosociales. Appelée Association des Aides Ados et Pairs Educateurs en Santé de la Reproduction à Saint-Louis et Association des Educateurs Communautaires de Santé à Louga, ils disposent d'un bureau logé dans une salle du centre de santé à Saint-Louis et au CDEPS du Ministère de la Jeunesse à Louga. Les membres de cette association maintiennent le lien avec leurs Organisations Communautaires de Base d'origine.

Le dynamisme de l'association des Pairs Educateurs de Saint-Louis se traduit par l'organisation régulière d'activités de masse lors des événements spéciaux comme les festivals de musique, la fête de l'Assomption le 15 août, la fête de l'indépendance le 4 avril, les journées SIDA et autres événements.

Les associations maintiennent aussi des liens étroits avec le district sanitaire qui les implique systématiquement dans les activités d'autres programmes tels que le Paludisme, le PEV, le SIDA, la Tuberculose. Ceci permet au district de les motiver économiquement et maintien le lien. Leur compétence et leur savoir faire sont fréquemment sollicités par d'autres partenaires au développement tels Plan International, Family Health International, Management Science for Health. Les deux associations ont bénéficié pour l'année 2005 de financements du Comité National de Lutte contre le SIDA (CNLS) à concurrence de montants de 2777450 de F. CFA pour Saint-Louis et 2834535 de FCFA pour l'association de Louga. La location des chaises données durant la recherche opérationnelle comme équipement générateur de revenus, et utilisé par les Pairs Educateurs pour leur motivation lors de l'expérimentation est restée une source de revenus à Louga. Les fonds provenant de la location de ces matériels servent de motivation interne pour les membres de l'association, à régler les cas sociaux et à fidéliser les membres. En plus, de ce système chaque membre de l'association cotise tous les mois, 1.000F CFA qui est mis dans une caisse commune.

Sur la base de besoins exprimés lors de l'analyse de la situation, plusieurs activités ont été menées à savoir : 
- Le recyclage des membres du Comité Technique Local de Saint-Louis qui a permis entre autres de discuter sur les processus d'extension et de pérennisation des activités, sur les capacités d'intégration de la Santé de la Reproduction des Adolescents dans les autres programmes de santé pour maximiser les possibilités de financement des activités d'éducation des jeunes et sur les moyens d'optimiser la synergie avec les activités de l'école (Inspection d'Académie, Infirmeries scolaires, Inspection Médicale des Ecoles) ;

- Le recyclage de l'association des pairs éducateurs de Saint-Louis qui a été l'occasion de revenir sur les techniques de communication et la méthodologie du curriculum Grandir en Harmonie, mais aussi de discuter de la question de l'intégration des activités de Santé de la Reproduction des Adolescents dans les activités des Pairs Educateurs, de l'extension de ces activités et de leur pérennisation, et enfin de la gestion administrative de leur association ;

- Des discussions et des suggestions de solution pour améliorer le mécanisme de recherche de financement et celui du renouvellement des pairs éducateurs.

Ces différentes activités ont permis de maintenir un engouement, de redynamiser, consolider et pérenniser les activités de santé des adolescents dans les deux régions expérimentales. Par ailleurs plusieurs facteurs ont permis d'améliorer la pérennité des activités de santé des adolescents dans ces sites.

A Saint-Louis et à Louga, les centres de santé se sont effectivement retrouvés au cœur d'un dispositif multisectoriel avec des liens très forts avec les services des Ministères de la Jeunesse, du Développement Social, de la Justice et aussi des organisations de jeunes. Ce partenariat fructueux constitue un acquis important de la recherche opérationnelle et a eu un impact important sur les populations :

\section{Implication soutenue des parents dans les activités de santé des adolescents}

A Saint-Louis, le partenariat autour de la Santé de la Reproduction des Adolescents est visible à travers l'extension des activités par le service du Développement Social qui poursuit ses séances d'animation envers les parents. Il est important de signaler que les opportunités de financement offertes par les autres programmes et institutions comme FISA, qui est une ONG Italienne, et le Comité Régional de Lutte contre le SIDA (CRLS), ont largement aidé à renforcer la synergie des activités concernant les adolescents à Saint-Louis.

Aussi bien à Saint-Louis qu'à Louga, des échanges réguliers continuent à se tenir dans la communauté entre les leaders religieux, les parents et le district sanitaire.

\section{Prise en charge holistique des cas d'abus sexuels}

A Louga, le Centre Conseil pour Adolescents du Ministère de la Jeunesse a été redynamisé par l'association des Pairs Educateurs et il existe un système performant de références entre ce Centre Conseil Ado, le centre de santé et le service de l'Action Educative en Milieu Ouvert (AEMO) du Ministère de la Justice. L'exemple de cette synergie est la mise en place d'un dispositif de prise en charge des abus sexuels sur le plan médical (par le district sanitaire), psychosocial (par le centre conseil ado) et médico-légal (par l’AEMO). 


\section{Renforcement des capacités des adolescents en rupture familiale et des adolescents travailleurs}

La collaboration fructueuse tissée entre le service de l'AEMO et CLAIRE ENFANCE qui est une ONG caritative française, se traduit concrètement par l'organisation de rencontres avec les jeunes incarcérés et délinquants dans les espaces d'éducation où ces jeunes mènent des activités de lecture, de couture, d'alphabétisation... Le curriculum Grandir en Harmonie est un support d'éducation de cette cible. En effet pour sensibiliser les enfants en situation difficile et les jeunes en danger (domestiques, cireurs, laveurs de voitures et filles mères) sur leur sexualité et les IST/SIDA, CLAIRE ENFANCE a sollicité l'assistance technique de l'AEMO de Saint-Louis qui disposait déjà de pairs éducateurs formés avec le curriculum Grandir en Harmonie pour utiliser le curriculum comme principal support pédagogique. CLAIRE ENFANCE dispose de groupes de base, composés de jeunes en danger, dans presque tous les quartiers de Saint-Louis envers lesquels elle mène des activités de prévention. Devant ces cibles différentes de celles généralement visées par les pairs éducateurs, CLAIRE ENFANCE et AEMO ont adopté une approche pédagogique du curriculum par rapport au milieu. Plus de 400 adolescents ont été touchés lors des séances de sensibilisation.

Un autre partenariat a été créé entre l'AEMO de Saint-Louis et une ONG américaine, Youth, Education and Sport (YES) permettant à l'AEMO d'utiliser le curriculum dans le cadre de la sensibilisation des jeunes pendant les camps de vacance organisés par YES. Trois éditions ont été déjà organisées depuis 2002 regroupant chacune 140 jeunes de la tranche d'âge de 12 à 18 ans.

\section{Intégration de la Santé des Adolescent(e)s dans les autres secteurs}

$\mathrm{Au}$ niveau central, des secteurs ministériels ont été influencés par différentes stratégies et approches mises en œuvre lors de la recherche opérationnelle sur l'amélioration de la Santé de la Reproduction des Adolescents.

\section{Intégration de la santé des adolescents dans les établissements scolaires}

Le Ministère de l'Education et son partenaire le GEEP ont entièrement intégré la santé de la reproduction comme une composante nécessaire à intégrer dans les activités de l'école. Le processus d'intégration se poursuit tout doucement, et il faut reconnaître que cela se fait dans l'esprit de la pérennité, car l'input de FRONTIERS est essentiellement de disséminer les leçons apprises, et de faire participer le Ministère de l'Education et le GEEP dans toutes les activités servant l'utilisation des résultats de la RO. La participation de ces structures dans l'élaboration de la Stratégie Nationale en Santé des Adolescent(e)s/Jeunes au Sénégal et dans la révision des curricula de formation permet de maintenir l'esprit et la volonté pour une approche multisectorielle et multidisciplinaire de la santé des adolescents.

Le Groupe pour l'Enseignement et l'Etude de la Population (GEEP) qui était partie prenante du volet des interventions à l'école lors de la recherche opérationnelle, continue à expérimenter le curriculum sur la santé des adolescents, élaboré lors de la recherche. Le curriculum le Devenir Accompagné, a été introduit dans deux lycées de la région de Thiès (à Thiès et à Mbour) et un lycée de la région de Louga (Dahra). Une deuxième phase est prévue à Dakar au Lycée des Parcelles Assainies, et à Mboro pour l'année 2006. Le curriculum Grandir en Harmonie va être utilisé pour les activités parascolaires telles que les camps de vacances avec d'autres supports tels que le manuel intitulé «Brisons le silence ». 
Le GEEP a pour autre projet de développer le modèle d'intégration des services de santé de la reproduction à l'école avec la restructuration de l'espace de santé scolaire, l'offre d'un paquet de services par une équipe pluridisciplinaire et des activités d'animation sur la santé des adolescents dans la salle du foyer du lycée. Un espace cyber et une salle d'écoute et de lecture feront partie de ce dispositif que constitue l'Espace Santé dans l'établissement scolaire. La phase pilote de ce projet devrait se dérouler au Lycée Blaise Diagne de Dakar dès la rentrée scolaire 2005/2006.

La Division du Contrôle Médical et Scolaire est en train de mettre en œuvre le projet d'Intégration de Services de Santé de la Reproduction à l'école en formant le personnel, les enseignants et les infirmiers scolaires avec le curriculum d'orientation des prestataires de santé sur la santé des adolescents, et en sensibilisant les parents et les élèves. Par ailleurs, ils ont utilisé le modèle de réorganisation des services de la Recherche Opérationnelle afin de rendre le Service Médical Scolaire plus agréables pour les adolescent(e)s/jeunes.

\section{Intégration de la santé des adolescents dans la formation des agents du Ministère des Sports}

Dans le processus d'intégration de la santé des adolescents dans la formation de leurs agents, le Ministère des Sports a sollicité la DSR et Population Council pour la formation de la promotion sortante des élèves maîtres d'Education Populaire et Sportive du Centre National d'Education Populaire et Sportive de Thiès. Ces élèves ont été orientés pendant deux jours sur la santé des adolescents avec le curriculum Grandir en Harmonie. Cette session a été l'occasion de discuter avec l'équipe d'encadrement du CNEPS des stratégies d'intégration de la santé des adolescents dans le cursus de formation des agents du Ministère et de l'appropriation par le Ministère des Sports des questions de santé des adolescents dans le cadre d'une approche multisectorielle de cette question telle que décrit dans la SNSA.

\section{Eléments pour un plaidoyer par le Ministère de la Jeunesse}

Le Projet de Promotion des Jeunes (PPJ) du Ministère de la Jeunesse a utilisé les résultats de la recherche pour élaborer un document de plaidoyer en direction des élus locaux afin de trouver des financements pour les activités de santé des adolescents.

\section{Extension dans les autres régions du Sénégal}

Une assistance technique a été apportée à la Division de la Santé de la Reproduction pour l'élaboration d'un plan d'actions en santé des Opérations qui a été soumis et approuvé par la Banque Mondiale par le biais de la Division de la Lutte contre le Sida (DLSI / Ministère de la Santé) et du Comité National de Lutte contre le SIDA (CNLS). Ce financement a permis d'entreprendre l'institutionnalisation de la santé des adolescents au Sénégal à travers :

- Un plaidoyer envers les partenaires au développement (UNFPA, UNICEF, Banque Mondiale...) a permis le financement de plan d'actions DSR/CNLS. La Division de la Santé de la Reproduction avec l'assistance technique de Population Council a élaboré un plan d'actions en santé des adolescents qui a été soumis et approuvé par la Banque Mondiale par le biais de la Division de la Lutte contre le Sida (DLSI et du Comité National de Lutte contre le SIDA (CNLS). Ce financement a permis d'entreprendre le passage à l'échelle de la santé des adolescents au Sénégal dans 9 des 11 régions du Sénégal à travers : 
> La dissémination des résultats de la RO sur l'amélioration de la SRA au Sénégal, la présentation de la Stratégie Nationale en Santé des Adolescent(e)s/Jeunes au Sénégal, l'élaboration de plans d'actions en santé des adolescents pour les districts sanitaires et les régions médicales et la création d'un Comité Technique Local pour la santé des adolescents dans les districts sanitaires ;

$>$ Une série de trois (3) sessions de formations des formateurs en santé et développement des adolescent(e)s/jeunes qui a été entreprise au Sénégal par le Bureau des Adolescents du Ministère de la Santé et de la Prévention Médicale avec les deux curricula de formation destinés aux prestataires de santé et aux pairs éducateurs. Au total, 100 formateurs ont été formés y compris des représentants d'OCBs de jeunes, notamment la section sénégalaise de l'ONG YMCA et Synergie Banlieue, des prestataires de santé, des agents des Ministères de la Jeunesse, de l'Education, de la Femme, de la Famille et du Développement Social, des partenaires au Développement...

$>$ Les formations de 120 prestataires de santé des régions touchées ;

$>$ Formation des Aides Adolescents: 33 Personnels jeunes ou Agents de Santé Communautaires intervenant dans les centres de santé des districts sanitaires de six régions médicales ont été formés avec le curriculum Grandir en Harmonie.

- Un renforcement des capacités du personnel de santé et des personnes intervenant dans le domaine de la santé des adolescents dans ces neufs régions. En effet, à la suite des formations de formateurs, la cascade des formations s'est poursuivie avec la formation des prestataires de santé dans 8 des régions touchées (Dakar, Thiès, Diourbel, Fatick, Kaolack, Saint-Louis et Louga). Un total de 120 prestataires a été formé avec le Module d'Orientation des prestataires de Santé en Santé des Adolescents ;

- La finalisation des outils de gestion des services cliniques offerts aux adolescents et des activités des pairs éducateurs sur le terrain. En effet, dans le contexte actuel de passage à l'échelle des activités de santé des adolescents dans les autres régions du Sénégal et pour la continuité des services dans ces régions, il est nécessaire de disposer d'outils de collecte des données sur les activités d'offres de services cliniques et de Communication pour un Changement de Comportement (CCC) destinés aux adolescents pertinents. Un atelier de révision des outils de gestion cliniques et du matériel promotionnel en santé des adolescents organisé en Septembre à Dakar a permis de :

> Sélectionner les outils de gestion des activités cliniques de santé de la reproduction des adolescent(e)/Jeunes les plus pertinents à intégrer dans le Système National d'Information Sanitaire (SNIS) ;

$>$ Simplifier et standardiser les outils de suivi et évaluation des activités des pairs éducateurs et des superviseurs sur le terrain ;

$>$ Finaliser les supports promotionnels existants et entamer la conception des nouveaux supports destinées aux cibles qui jusque là n'avaient pas été pris en compte (telles que les parents, les enseignants, certaines catégories d'adolescents...).

- L'intégration d'indicateurs de la Santé de la Reproduction des Adolescents dans SNIS est acquise. En effet, le processus d'harmonisation des indicateurs de la Santé de la Reproduction entrepris par la Division de la Santé de la Reproduction du Ministère de la Santé et de la Prévention Médicale a pris en compte la cible des adolescents et des jeunes pour lesquels trois indicateurs clés ont été retenus, à savoir :

$>$ Le Taux de fréquentation des structures de santé par les adolescent(e)s/Jeunes ;

$>$ La disponibilité de structures adaptés pour les adolescent(e)s/Jeunes, et

$>$ La capacité d'auto prise en charge des adolescent(e)s/Jeunes. 
Aujourd'hui, ces neuf régions disposent de plans d'actions pour la santé des adolescents, de même que des formateurs. Avec l'assistance du Ministère de la Santé et l'appui financiers de leurs partenaires respectifs, ces régions devraient pouvoir mettre en œuvre et prendre en charge correctement des activités de santé des adolescents.

\section{Utilisation d'outils élaborés ou des éléments du Modèle de la recherche opérationnelle par d'autres partenaires au développement}

- Le Ministère de la Jeunesse à travers de Projet de Promotion des Jeunes et le Ministère de la Jeunesse à travers la Division de la Santé Scolaire ont reproduit le curriculum pour l'utiliser dans la mise en œuvre de leur programmes respectifs financés dans le cadre du $6^{\text {ème }}$ programme d'assistance de UNFPA au Sénégal. Leurs agents avaient été formés lors de la première session de formation des formateurs. Par ailleurs, la Direction de la

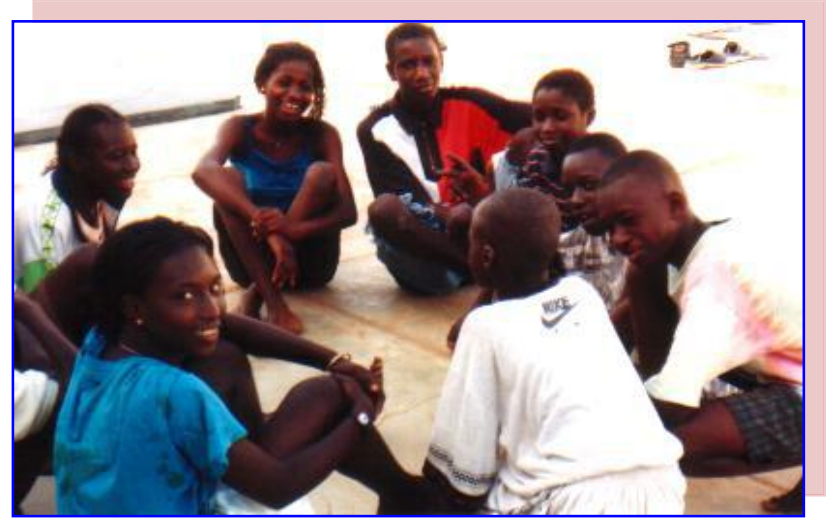
Jeunesse et de la Vie Associative (DJVA) utilisent le curriculum Grandir en Harmonie pour la formation des Pairs Educateurs ;

- Management Sciences for Health (MSH) a pris en compte la Santé de la Reproduction des Adolescents dans la formation des enseignants en 2003 avec le curriculum développé lors de la recherche opérationnelle. Au total, 244 Inspecteurs d'école ont été formés en Santé de la Reproduction des Adolescents avec, entre autres outils de formation, le curriculum «Le Devenir Accompagné ». Par ailleurs, les pairs éducateurs à Louga ont joué un rôle important dans la mobilisation sociale ;

- Le bureau régional de l'UNICEF a recommandé l'utilisation du curriculum Grandir en Harmonie dans d'autres pays de la sous région tel que le Burkina Faso et le Bénin. Par ailleurs, ce bureau organise un atelier régional sur Jeunes et VIH/SIDA qui devrait se tenir à Dakar en Décembre 2005, réunissant les 15 pays couverts par le bureau de l'UNICEF. Le modèle de services conviviaux pour les jeunes intéresse particulièrement le Bureau régional de l'UNICEF qui souhaite utiliser les membres de l'équipe de FRONTIERS comme personnes ressources lors de cet atelier ;

- $\boldsymbol{C E D P A}$ en collaboration avec Population Council, et au regard des résultats de la recherche opérationnelle sur la Santé de la Reproduction des Adolescents, le Centre pour le Développement et les Activités de Population (CEDPA) a mis en œuvre, dans la région de Kaolack, un projet sur l'Amélioration de la Communication sur la Santé de la Reproduction dans les familles d'adolescent(e)s au Sénégal. Les résultats de cette étude ont fourni un aperçu considérable de l'expérimentation sur la communication entre les parents et les adolescents. Ces résultats ont été intégré dans le document de politique de l'OMS intitulé WHO parenting project et seront utilisés par les Ministères de la Santé, de la Jeunesse, l'UNICEF, USAID, UNFPA, Plan International et l'ASBEF ; 
- L'UNICEF et l'UNFPA : dans la mise en œuvre conjointe du projet de renforcement des capacités des adolescentes défavorisées, le questionnaire de la recherche opérationnelle a servi de modèle de base pour l'élaboration du questionnaire d'évaluation dudit projet ;

- L'UNFPA a accepté d'utiliser le modèle d'amélioration de la santé de la reproduction des adolescents dans quelques districts de leurs zones d'interventions ;

- ADEMAS qui est l'Agence pour le Développement du Marketing Social est en train de mettre en œuvre un projet qui a pour cible les adolescents de 15 à 24 ans. Ce projet comprend trois principaux volets: la Communication pour un Changement de Comportement, la mise à disposition du préservatif pour les adolescent(e)s et la promotion du préservatif féminin. ADEMAS est particulièrement intéressée par la communication entre parents et adolescent(e)s, l'identification et l'installation de services et la disponibilité de prestataires conviviaux pour les adolescent(e)s/jeunes ;

- APAPS: dans le cadre de la mise en œuvre d'un projet sur Jeunes et VIH/SIDA, L'Agence pour la Promotion des Activités de Population (APAPS) utilise le curriculum Grandir en Harmonie pour la formation de ses pairs éducateurs. L'association des Aides et Pairs Educateurs en santé de la Reproduction de Saint Louis qui était l'un des sous récipiendaire de l'Agence pour la Promotion des Activités de Population (APAPS ) a reçu un montant de quatre cent vingt mille francs dans le cadre de la mise en œuvre du Programme du Fonds Mondial exécuté par l'Alliance Nationale de Lutte contre le SIDA. Vingt quatre (24) séances de causeries ont été réalisées par les Pairs Educateurs durant le dernier trimestre de l'année 2007. Durant ces séances qui ont touchées 176 personnes (95 hommes et 81 femmes) âgées de 15 ans à 35 ans, des préservatifs (condom et fémidon) ont été distribués aux participants qui le désiraient ;

- Save the Children Suède, compte tenu de l'impact du VIH/SIDA sur la vie des enfants et $\mathrm{du}$ taux d'infection au niveau des jeunes, a décidé d'intégrer la problématique du VIH/SIDA dans son programme régional, à partir de 2004. Ce programme met surtout

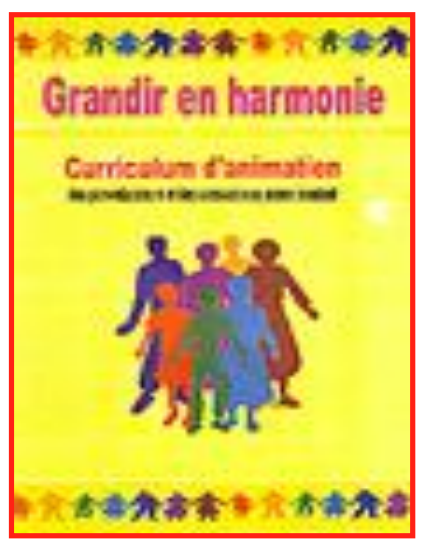

l'accent sur la prévention de l'infection au niveau des adolescents à travers l'information et l'accès aux services de santé de la reproduction. Ayant identifié le curriculum «Grandir en Harmonie » comme étant un outil très efficace qui peut contribuer à l'atteinte de ses objectifs, SCS a demandé 20 exemplaire du curriculum destinés à ses partenaires. Par ailleurs, SCS est disposée à renforcer la collaboration avec Population Council pour mieux outiller les ONG dans la sous région en matière de santé de la reproduction des adolescents et deux proposals sont en cours d'élaboration dans ce sens.

- Les organisations Communautaires de jeunes dont les membres ont été formés ont aussi été renforcées en curriculum. Et ces pairs éducateurs ainsi formé sont en train de mener des séances d'éducation en compétences de vie à l'aide du curriculum Grandir en Harmonie auprès d'autres jeunes ;

- L'ONG YMCA est présente au Sénégal depuis 1982 dans le domaine du développement avec comme principales cibles les jeunes, les femmes et les couches très vulnérables de la communauté. Elle intervient dans cinq régions du Sénégal (Dakar, Thiès, Saint-Louis, 
Kaolack et Ziguinchor) dans les domaines de la Santé, de l'Entrepreunariat/Développement, la Jeunesse, l'alphabétisation, de l'InfoNet Jeunes et dispose d'un Centre de Formation Professionnelle aux Parcelles Assainies, d'un Domaine dans les Niayes et d'un Groupe Scolaire. Elle est structurée en un Bureau National dirigé par le Secrétaire Exécutif National, des commissions nationales, des Unions Régionales et des branches régionales. En tant que structure d'encadrement national du Conseil National de Lutte contre le SIDA (CNLS) dans le cadre du projet SIDA, YMCA/Sénégal a sollicité l'assistance technique de Population Council pour un renforcement de capacités en Santé de la Reproduction des Adolescent(e)s et VIH/SIDA.

Six branches de YMCA/Sénégal ont mené des activités d'éducation avec le curriculum. Les branches sont les démembrements de YMCA au niveau local. Six autres Organisations de Jeunes qui sont des partenaires de l'YMCA/Sénégal dans le domaine de la Santé de la Reproduction \& VIH/SIDA ont aussi utilisé le curriculum Grandir en Harmonie. Il s'agit de Cœurs Vaillants Ames Vaillantes (CVAV) de Ste Anne, Association des Relais Polyvalents (ARPV), CVAV de Thialy, les Jeannettes de Ste Anne, les Guides et Eclaireurs de Ste Anne et les Pionniers. Au total, 5693 filles et 2626 garçons ont été touchés.

- Créée en 2003 grâce à une initiative locale de jeunes, Synergie Banlieue est une association qui intervient principalement dans quatre (04) communes d'arrondissement (Pikine-Ouest, Pikine-Nord, Pikine-Est et Pikine-Guinaw Rail). Après la formation de leurs membres avec le curriculum Grandir en Harmonie, Synergie Banlieue l'a utilisé pour renforcer ses actions en faveur de la santé des adolescents à travers deux axes majeurs à savoir le renforcement des capacités du Réseau des Jeunes Volontaires de la Santé de Pikine (RJVS/P) et le renforcer les services du centre Synergie et les actions en direction des adolescents déscolarisés et vivant dans des

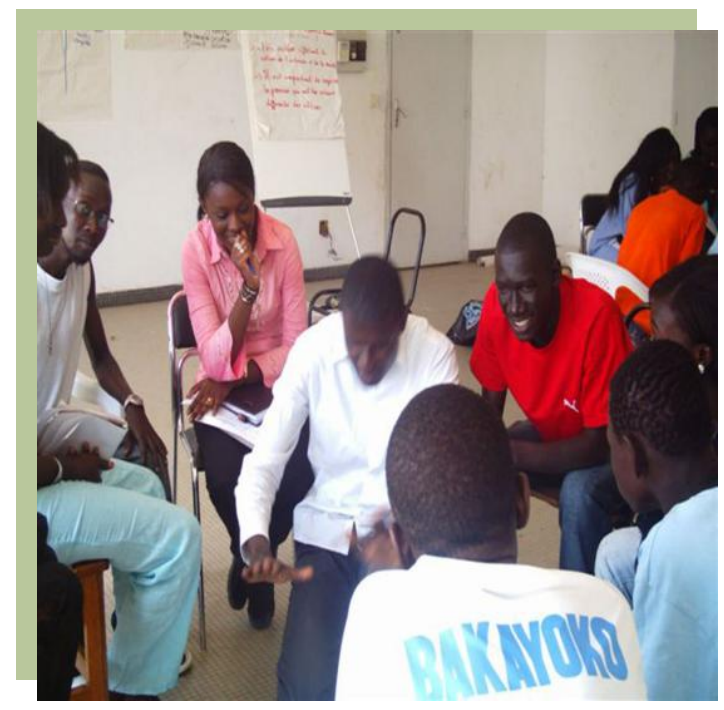
conditions difficiles comme les enfants des rues.

- Le Réseau des Jeunes Volontaires de la Santé de Pikine (RJVS/P) est une structure fédératrice mise en place par Synergie banlieue pour promouvoir la participation des adolescents/ dans la lutte contre le SIDA. Elle est composée de quatre entités regroupant chacun 25 jeunes âgé(e)s entre 15 et 22 ans et présents dans les communes de PikineOuest, Pikine-Est, Pikine-Nord et Guinaw-Rail. Il bénéficie de la couverture institutionnelle et de l'assistance technique de Synergie banlieue dans la mise en œuvre, le suivi des activités et de l'appui financier de certaines structures d'appui aux organisations de base comme l'Alliance Nationale Contre le Sida (ANCS). 20 jeunes animateurs du Réseau (dont 08 filles et 12 garçons) issus de quatre communes d'arrondissement de Pikine ont été formés dans le but de pouvoir mener efficacement des activités d'éducation et d'animation en santé de la reproduction auprès de leurs pairs à l'aide du curriculum. Après la formation des animateurs 10 pairs éducateurs membres ont 
été sélectionnés comme animateurs-éducateurs principaux. Les pairs éducateurs du Réseau ont utilisé le curriculum pour animer des séances d'éducation en santé de la reproduction au niveau à Pikine. Ce programme d'une durée de deux mois a bénéficié d'une subvention de l'ANCS. Sept séances d'animation ont été organisées auprès de 165 adolescents de Pikine âgés(es) entre 12 et 18 ans dont 72 filles et 93 garçons. Parallèlement, des séances d'animation ont été programmées au centre avec le Curriculum «Grandir En Harmonie ». L'objectif était de compléter les activités d'information et de conseil par des séances destinées à accroître les connaissances et les compétences des adolescents. Au total 20 séances « life skills » ont été organisées par la conseillère du centre avec 345 adolescents âgés entre 14 et 18 ans.

Un partenariat a été noué avec les structures intervenant spécifiquement auprès d'enfants non scolarisés, jusque là insuffisamment touchés par les actions prévention du VIH/SIDA ou de promotion de la SR. Il s'agit d'accroître les connaissances et les compétences de ces adolescents en SR à travers des séances d'animation à partir du curriculum Grandir en Harmonie. Synergie Banlieue a signé deux conventions de partenariat avec deux ONG travaillant auprès de ces enfants.

- Le Village pilote : une ONG française établie au Sénégal et qui intervient dans la prise en charge et la réinsertion des enfants des rues ;

- L’Association Nationale des Handicapés moteurs du Sénégal.

Synergie Banlieue prévoit d'élargir ces séances d'animation en SR vers les Centres d'Enseignement Technique féminins qui accueillent en majorité des filles exclues du système scolaire classique. Dans cette perspective, un accord de partenariat devrait être établi avec le centre Social de Pikine, le Centre de Perfectionnement Technique Féminin de la Cité Sotiba et le Collectif pour l'Education Alternative (CEA) qui regroupe plus de 50 Ecoles Communautaires de Base (ECB) installées principalement dans la zone de Guinaw-Rail.

- Traduction du Curriculum en Arabe et Formation des Formateurs en Mauritanie 26 Pairs Educateurs ont été formés avec le curriculum Grandir en Harmonie qui a été adapté et traduit en arabe dans le cadre du programme de Prévention du VIH/SIDA du bureau de l'UNICEF en Mauritanie.

\section{Facteurs de réussite de l'utilisation des résultats de la recherche opérationnelle}

Plusieurs facteurs ont contribué à maintenir le dynamisme autour des activités de santé des adolescents au Sénégal, notamment :

- Le rôle clé du Ministère de la Santé dans la mise en œuvre des activités de santé des adolescents et la création d'outils de formation nationaux à travers notamment la création d'un groupe synergique pour impulser, coordonner, consolider et pérenniser les activités de santé des adolescents a permis aux différents acteurs de pouvoir échanger, discuter et planifier les activités a été cruciale. L'existence de ce groupe a nourrit le long processus consultatif qui a permis d'aboutir à la révision du curriculum Grandir en Harmonie complété par deux nouveaux documents: le guide de l'utilisateur et les questions/réponses les plus fréquentes posées par les adolescents. Le curriculum 
d'animation et le document de références ont été améliorés avec l'introduction de nouvelles sections tels que le Conseil et le Dépistage Volontaire (CDV), des étapes d'évaluations, de nouveaux jeux sur le SIDA.... Dans le même processus les curricula de formation en santé des adolescents pour les prestataires de services et pour les jeunes (Grandir en Harmonie) ont été approuvés comme documents nationaux en Juin 2005. L'ensemble de ces documents a servi à la formation des prestataires de santé et des jeunes au Sénégal ;

- L'approche multisectorielle a permis l'implication et l'engagement de plusieurs secteurs ministériels et la communauté mettant en exergue l'importance de la santé et du développement des adolescents comme une priorité politique et sociale. Par ailleurs, l'engagement ferme de l'OMS a aussi permis d'impulser cette approche multisectorielle ;

- La satisfaction des besoins des populations. Les résultats de la Recherche Opérationnelle ont été primordiaux pour pérenniser les activités de santé des adolescents dans la mesure où ces activités ont eu pour base une méthode scientifique d'investigation de la question. En outre, le projet à répondu au besoin des gestionnaires de programmes, des communautés et les parents ont été conscient du besoin de prendre en charge la santé et le développement des adolescents, ce qui a faciliter leur engagement ;

- Un des principes directeurs de la recherche sur la Santé de la Reproduction des Adolescent(e)s était de bâtir la pérennisation à travers l'utilisation des structures existantes au lieu d'en créer de nouvelles. Par exemple, le partenariat entre les associations de jeunes et les structures de santé publiques a induit un fort engagement des pairs éducateurs et leur maintien dans le programme. Les pairs éducateurs qui ont été formés durant la phase pilote du projet sont devenus des formateurs pour les nouveaux groupes de pairs éducateurs.

\section{Facteurs contextuels ayant limité l'utilisation des résultats de la recherche}

- Le manque de disponibilité de financements pour les activités de santé des adolescents avec notamment les phases de fin de programmes de l'USAID, de l'UNFPA et de GTZ qui se sont terminé durant le travail sur les mécanismes de l'utilisation. Ceci a limité les possibilités de financement ;

- D'autres financements pour les activités de santé des adolescents sont aussi très faiblement disponibles au niveau décentralisé des Ministères de la Jeunesse et du Développement Social, malgré les requêtes du niveau local ;

- L'intégration insuffisante de la Santé de la Reproduction des Adolescents dans les autres programmes du Ministère de la Santé. En effet la santé des adolescents est transversale et sa définition comporte huit composantes parmi lesquelles la maternité sans risque, la planification familiale, les IST et le VIH, la sexualité etc. Au niveau des deux districts d'expérimentation, son intégration n'est pas totalement acquise au niveau de ces programmes. Les différents acteurs ont du mal à percevoir les adolescents comme étant une cible qui pourrait être prise en charge dans la mise en œuvre de ces programmes verticaux, en utilisant le dispositif expérimenté sur la Santé de la Reproduction des Adolescents. En effet, les adolescents étant une des cibles prioritaires des IST/VIH/SIDA, de la maternité sans risque et d'autres programmes, une telle approche contribuerait à l'atteinte des objectifs de ces programmes. Cette non intégration des activités de Santé de 
la Reproduction des Adolescents dans les autres programmes a pour conséquence de ne freiner les activités d'éducation des adolescents à cause du manque de financement pourtant disponible à travers ces programmes. C'est le cas particulièrement des activités de proximité telles que les animations dans les quartiers avec le curriculum Grandir en Harmonie qui ont été ralenties ;

- Le processus de révision et de validation officielle des documents des Ministères (normes et protocoles, orientations stratégiques, SNIS) a été long ;

- Un changement de personnel clé à la Division de la Santé de la Reproduction du Ministère de la Santé.

\section{CONCLUSION}

L'utilisation des résultats de la Recherche Opérationnelle sur l'Amélioration de la Santé de la Reproduction des Adolescent(e)s a permis de maintenir et même d'accroître l'intérêt et le dynamisme pour la santé des adolescents. Aujourd'hui, le Sénégal, à travers le Ministère de la Santé et de la Prévention Médicale dispose de tous les éléments de base pour l'appropriation et l'extension des activités de santé et de développement des adolescent(e)s dans toutes les régions. En effet :

- La Stratégie Nationale en Santé des Adolescent(e)s/Jeunes permet de guider les districts sanitaires et leurs partenaires dans la mise en œuvre des activités ;

- Les fiches techniques leur permettent de planifier les activités prioritaires et de les budgétiser ;

- Les outils de formation des prestataires de santé et des pairs éducateurs communautaires sont disponibles ;

- Un pool de 100 formateurs aux niveaux national et régional a été constitué et est déjà actif ;

- Le curriculum et les outils de formation des enseignants et des LEA à l'école sont disponibles ;

- Au niveau central, une équipe multisectorielle et pluridisciplinaire est disponible pour accompagner les régions médicales et les districts sanitaires dans le processus.

Aujourd'hui 9 des onze régions que comptent le Sénégal ont impulsé les activités de santé des adolescents et sont à des niveaux différents dans la mise en œuvre de ces activités. Plusieurs défis restent à soulever :

- Le financement des activités de santé et de développement des adolescents par le gouvernement du Sénégal à travers les budgets des différents Ministères qui ont en charge cette question, à travers les budgets disponibles au niveau local des régions et par les partenaires au développement ; 
- Le renforcement de la multisectorialité et du partenariat par la mise en œuvre conjointe avec tous les acteurs impliqués de la Stratégie Nationale en Santé des Adolescent(e)s/Jeunes ;

- La promotion des leçons apprises et l'utilisation des meilleurs pratiques communautaires pour prendre en charge la santé et le développement de adolescent(e)s parmi la communauté des agences de collaboration, les bailleurs, les agences de coopération internationale, les gouvernements e t les ONGs travaillant en Afrique de l'Ouest ;

- Le renforcement des capacités des institutions d'offre de service afin qu'elles puissent prendre le lead dans le passage à l'échelle d'un programme multisectoriel pour améliorer la santé des adolescent(e)s en Afrique francophone sur la base des leçons apprise de la recherche opérationnelle.

L'impact de ces activités sera d'accroître la mise en œuvre de programme multisectoriel pour les adolescent(e)s à travers l'Afrique de l'ouest. Impact qui sera démontré par les changements effectués par les groupes ciblés en termes d'orientation politique et programmatique et aussi de mise en œuvre des services pour adolescent(e)s. 